\title{
L'évolution des systèmes de prévision des crues et des inondations : de l'offre technique vers une offre de service au citoyen. Réflexions et perspectives
}

\author{
The evolution of flood forecasting : from the technical \\ offer to a citizen oriented services offer
}

par Marc Erlich et Patrick Sauvaget

SOGREAH

et Guy Taliercio

Consultant, Centre Equation

A prospective outlook on the development of crisis prevention, forecasting and flood management systems in flooding situations is proposed. Such systems were initially focused on technical expertise and then progressively widened to encompass the requirements of the technical user and, subsequently, the citizen, client or final user. The authors consequently take a look at potential services that could be offered, with possible deregulation, within the context of social requirements accompanying the sustainable development of the "Information Society ".

\section{I —UN SCÉNARIO POUR DEMAIN}

Imaginons un scénario pour demain...

Dans le bassin versant d'un fleuve (possédant une surface supérieure à $3000 \mathrm{~km}^{2}$ ) après une longue période de pluies d'intensités faible et moyenne (disons $200 \mathrm{~mm}$ en 45 jours) des précipitations d'une intensité forte $(300 \mathrm{~mm}$ en 24 heures) centrées sur la partie amont du bassin donnent lieu à des crues rapides qui se propagent ensuite dans la partie aval. De nombreux débordements sont constatés et l'onde de crue se déplace vers des zones particulièrement vulnérables. L'administration chargée de l'annonce des crues émet successivement des avis de vigilance, pré-alerte et alerte. Un état d'urgence est déclaré et les informations sont diffusées à la radio et à la télévision. Il y a rupture de l'alimentation en eau potable et en électricité, des réseaux de téléphonie commutés cessent de fonctionner, de graves problèmes de circulation se multiplient...

Jusque-là, rien de nouveau...

Imaginons que, dans une zone à risque, l'alerte soit transmise à chaque habitant qui en a fait la demande en s'inscrivant simplement sur la base de données du serveur des collectivités locales. Lors de son inscription, il a notamment précisé les localisations pour lesquelles il désire être alerté, la nature de la vulnérabilité, ses deux numéros de téléphone (dont celui de son terminal portable), le niveau à partir duquel il désire être alerté.

Une fois alerté, le riverain navigue avec les touches de son terminal : il consulte la météo locale des prochaines 24 heures, la carte des zones sur sa commune avec la prévision de hauteur d'eau sur 12 heures. Il peut déclencher une animation qui lui permet de voir l'évolution de la montée des eaux et lui indique par un voyant rouge clignotant que le point bas sur la route sera immergé dans une dizaine d'heures. Ne pouvant se déplacer dans le délai nécessaire, il consulte la base de données qui lui donne la liste des riverains de sa zone. Il sélectionne le nom de son voisin $Z$, ce qui a pour effet de le mettre en communication téléphonique (réseau mobile) immédiate avec lui. Il lui demande de bien vouloir s'occuper de l'évacuation des dix génisses qu'il a laissé paître sur la zone. Dans le but de limiter les dégâts d'eau probables dans son atelier on lui indique également les moyens les plus simples pour mettre à l'abri son matériel. Plus tard, se rendant sur place, il en profitera pour lire la hauteur d'eau sur le repère de zone et communiquer cette 
information au centre de gestion de crise à l'aide de son terminal portable. Cette information, recoupée avec d'autres, contribuera à la mise à jour des prévisions et de la cartographie dynamique élaborée par ce centre...

Vous avez dit science-fiction?

\section{II — POURQUOI UN TEL SCÉNA- RIO?}

Examinons la plausibilité d'un tel scénario à la lumière de la situation actuelle et de l'évolution à la fois de la demande sociale en matière d'accès à l'information compte tenu du développement technologique.

\subsection{L'évolution de la demande sociale}

\subsubsection{Expérience de la crue 1997 en Pologne}

En juillet 1997, lors de la crue catastrophique qui a frappé la Pologne, la République Tchèque et l'Allemagne, un phénomène sans précédent a eu lieu dans l'espace de communication offert par Internet. En réponse à un besoin pressant d'information sur l'évolution de la situation dans les parties inondées à l'échelle locale et régionale, une centaine de sites dédiés a été créée en Pologne (http://www.gnet.pl/powodzwroclaw/links/links.html). Selon la période de la crise, ces sites avaient une fonction de diffusion d'informations que l'on peut classer de la manière suivante (fig. 1) :

- Appels au secours (demande d'intervention urgente des services médicaux, demande de médicaments, vaccins, etc...).

- Appels à l'aide des bénévoles (pour le renforcement et la surveillance des digues, pour participer aux actions de sauvetage du patrimoine culturel : musées, bibliothèques universitaires).

- Demandes de renseignements sur les personnes habitant les zones inondées.

- Liste des personnes évacuées avec une localisation précise des centres d'hébergement.

- Communiqués divers (météo, photos, cartes des zones inondées, consignes de sécurité et de prévention des risques d'intoxication, informations routières, etc.).

- Appels aux porteurs de téléphones mobiles pour qu'ils transmettent des informations relatives à la situation sur le terrain (commune par commune, rue par rue).

- Appels aux services de police et à l'armée pour protéger les magasins contre le pillage et les actes de vandalisme.

- Appels aux administrateurs d'autres sites pour reprendre le relais des serveurs coupés d'électricité.

- Appels à l'aide humanitaire.

Rappelons en premier lieu que, dans la majorité des régions touchées, la crise a été consti-

1. Exemple de la page Internet "Flood in Poland " du site Virtual Poland (http ://wp.cnt.pl/Powodz/).

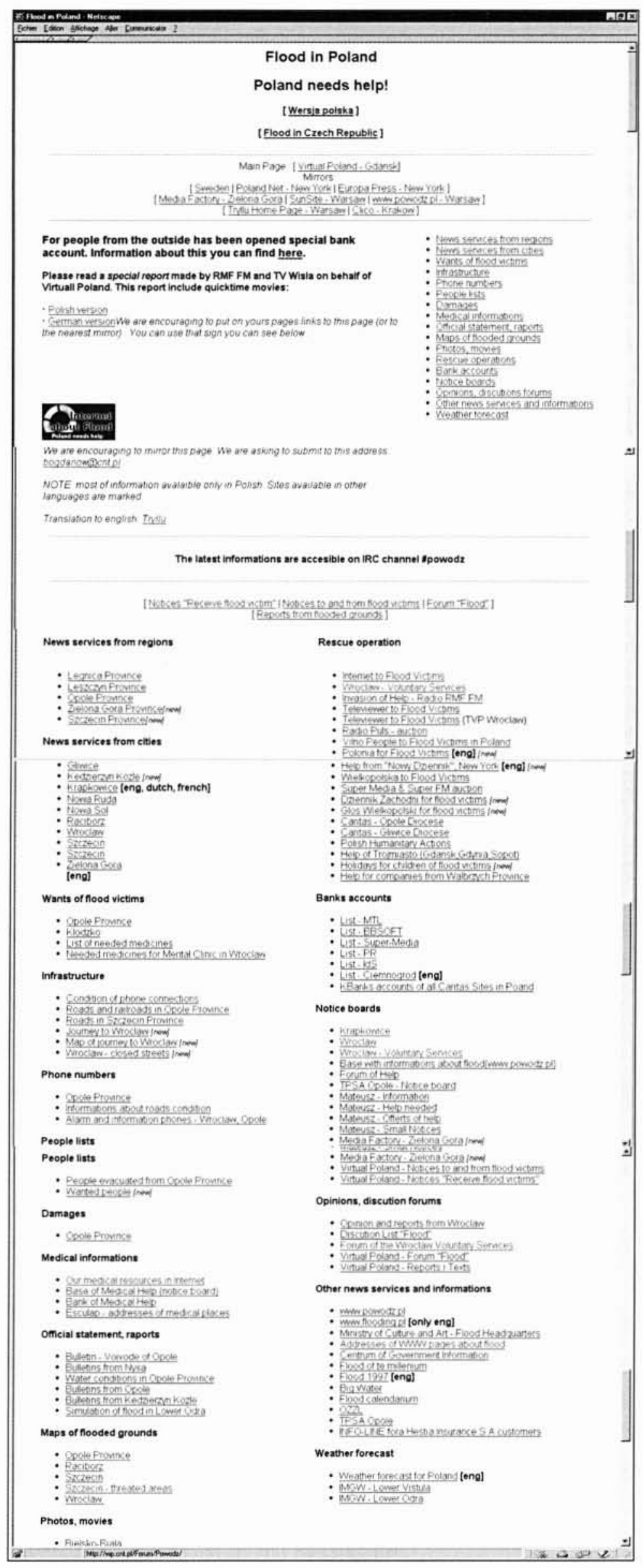


tuée de deux pointes successives, séparées de quelques jours. Une première crue rapide due à des précipitations intenses localisées sur la chaîne de Karkonosze précédées par une période préparatoire d'une durée de 15 jours d'une pluie généralisée a surpris les services de l'état et la population située dans les bassins de la Haute Odra (Oder), de la Nysa Klodzka et de la Vistule. Une deuxième crue, beaucoup plus lente, a permis l'organisation des secours et la préparation des riverains. La mise en service de serveurs automatiques d'insertion de messages et de forums de discussion a permis une très large diffusion de l'information alors que les communications téléphoniques ne fonctionnaient pas et que les autorités chargées de l'organisation des secours étaient complètement dépassées par les événements.

Il faut noter une importante participation de la communauté des internautes étrangers à cette action spontanée. Souvent bilingues (l'émigration polonaise compte quelques 10 millions de membres), ils facilitaient les tâches de traduction des pages Web élaborées par les serveurs polonais. Pour les habitants d'autres régions de Pologne que celles sinistrées ou pour ceux vivant à l'étranger à la recherche de nouvelles de leurs proches, ces sites ont constitué la seule source d'information étant donné que ni les services de la sécurité civile ni ceux de la Croix Rouge n'étaient accessibles.

Des sites miroirs ont fait leur apparition dans les pays voisins qui ont été également touchés par la crue (http ://www.grendel.cz/zaplavy/poland.html).

L'information recueillie sur ces sites a été analysée et a permis de constituer des cartes d'inondation à l'échelle des villes et des quartiers ; leur actualisation heure par heure permettait d'identifier les zones déjà inondées, les zones à risque et les consignes aux automobilistes avec indication des axes de circulation (fig. 2).

Cette expérience unique d'initiative spontanée de la société civile mérite une analyse plus profonde que celle que l'on peut offrir dans le cadre limité de cette communication. Notons également les bénéfices indirects de cette action, surtout pendant la période qui a suivi la crue :

- Internet a permis de structurer d'une manière cohérente l'aide humanitaire, la collecte des fonds, la reconstruction et la restructuration de l'activité économique dans les zones sinistrées.

- Les sites dédiés ont contribué à une prise de conscience débats qui ont suivi la catastrophe ont été chargés d'une sur la problématique des risques d'inondation. Certes les

forte composante socio-politique, mais progressivement la discussion s'est déplacée vers la recherche de solutions techniques ayant pour but d'améliorer la gestion de la crise à l'échelle locale.

- La crue de 1997 a mis en évidence la fragilité du système de communication entre tous les acteurs impliqués dans la problématique : météorologues, hydrologues, sécurité civile, armée, sans parler de la communication entre le Centre National de Lutte contre les Inondations et la population.

- Le manque d'information que les autorités auraient dû diffuser a amplifié l'importance des médias (surtout les stations de radio et de télévision locales) qui diffusaient des informations $24 \mathrm{~h} / 24 \mathrm{~h}$, mais souvent sans donner une vision globale du problème.

- Il est difficile de négliger la présence sur Internet des nombreux articles décrivant différents aspects des problèmes socio-économiques de la population sinistrée.

L'expérience vécue du fonctionnement des sites Internet pendant et après la crue a permis de mettre en évidence un certain nombre d'aspects négatifs, qu'il faudrait analyser de manière plus fine pour en tirer les conclusions définitives : - La diffusion d'informations erronées, que cela soit de manière intentionnelle ou par mégarde.

- Les forums de discussion ont donné l'occasion de débats marqués par la passion politique (recherche de coupables institutionnels responsables de l'annonce et de la gestion de la crue).

- Le manque de références techniques et l'improvisation dans l'explication des origines de la crue - la lecture de certains de ces messages montre que les techniciens (hydrologues, météorologues, psychologues, etc...) n'ont pas pris une participation active dans ces forums.

- Une utilisation détournée d'Internet à des fins commerciales (une multitude d'annonces proposant une aide gratuite aux victimes des inondations a été accompagnée par des listes de prix " très intéressants " pour les autres...).

\subsubsection{Initiatives aux Etats-Unis}

Aux Etats-Unis les dommages liés aux ouragans, tempêtes et crues représentent en moyenne un coût annuel de l'ordre de 10 milliards de dollars. Depuis 1996, dans le but d'améliorer la communication entre les chercheurs et praticiens de la prévision du temps d'une part et la société civile d'autre part, la National Oceanic and Atmospheric Administration (NOAA) et le National Center for Atmospheric Research (NCAR) financent un projet expérimental appelé «U.S. Weather Research Program (USWRP) ».

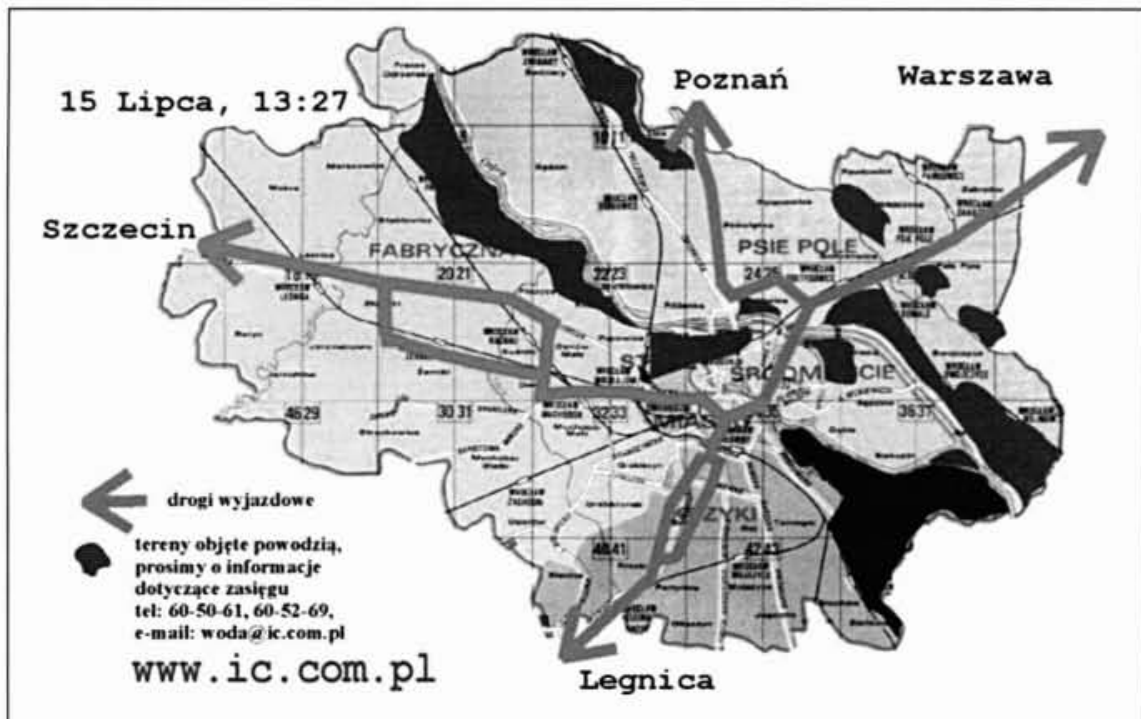

2. Cartographie des zones inondées de Wroclaw disponible sur Internet lors de la crue du juillet 1997. tique a été créé (http://www.dir. ucar.edu/esig/socasp/zine/) et ses sections thématiques consacrées aux problèmes spécifiques des inondations, des foudres et de l'agriculture contiennent des informations pratiques (conseils de simples règles de sécurité, actions préventives en cas d'alerte, etc...). Ce programme ayant une vocation pédagogique s'adresse également à un très large public (écoles, personnes privées, sociétés,...) par une sensibilisation aux différents risques liés aux phénomènes météorologiques qui frappent le continent nord-américain (crues rapides, tornades, etc.).

Une autre initiative orientée vers la diffusion de l'information en situation d'urgence a été mise en place dans le cadre des associations de
Un site Web dédié à cette probléma- 
bénévoles nord américains (des organisations et des individus), nommées « Emergency Information Infrastructure Partnership » (EIIP, http ://www.emforum.org) et HazardNet (http ://hoshi.cic.sfu.ca/hazard), qui cherchent à mettre en valeur la possibilité de partager l'information dans les situations de catastrophes et d'urgences via l'Internet. Elles tentent de démontrer que ces outils de communication globale ont leur place dans les actions de l'administration et des organismes impliqués professionnellement dans la lutte contre des situations de crise provoquées par des catastrophes naturelles et technologiques. Les partenaires actifs de cette initiative se recrutent aussi bien dans les services de l'administration que dans l'industrie, les universités ou centres de recherche et les ONG.

\subsection{Demande sociale et possibilités émergentes en France}

Trois éléments nous paraissent révélateurs de l'évolution de la demande sociale autant que de la pertinence des réponses pouvant être apportées :

- La logique de service.

- L'implication des citoyens et les pratiques de réseau.

- Le partenariat entre l'Etat, les collectivités locales et la société civile.

\subsubsection{Logique de service}

La commission d'enquête parlementaire constituée en 1994 mettait en évidence dans son rapport [1] la nécessité de mieux satisfaire les besoins d'information des communes riveraines de cours d'eau. Elle relevait le besoin d'améliorer la qualité de l'information, vers une information plus localisée, mieux interprétable par son utilisateur élu et riverain, assortie d'un délai de mise à disposition et d'une anticipation (prévision) adaptés au contexte local et au danger. Il s'agissait donc essentiellement d'une définition de l'information requise qui permet dans un deuxième temps de valider ou d'améliorer les processus d'acquisition des données, d'élaboration et de circulation de l'information (voir site de la DIREN Rhône-Alpes, Délégation de bassin RMC http ://rdb.eaurmc.fr/risques/html/Risques.html). Cette approche, basée sur la satisfaction de l'utilisateur final de l'information, met en avant la logique de service aux usagers. Dans une telle perspective, l'amélioration des infrastructures et des moyens de mesure, de prévision et d'alerte se déduit des besoins. C'est l'offre de service qui guide l'offre technique, et non l'inverse.

\subsubsection{Implication et responsabilisation des citoyens dans une perspective de développement durable - Pratiques de réseau}

L'implication des citoyens dans la prévention et la gestion des inondations est un des enjeux majeurs de la mise en œuvre des principes d'un développement durable dans le domaine de l'eau.

Cette implication se concrétise notamment en France, par le droit des citoyens (Art. 21 de la Loi du 22/07/87) à l'information sur les risques auxquels ils sont exposés [2]. Une directive européenne [3] décrit l'obligation faite aux autorités publiques des Etats membres de «mettre les informations relatives à l'environnement à la disposition de toute personne physique ou morale qui en fait la demande... ".

Cette obligation comporte toutefois une limite, exprimée par le fait que les Etats membres puissent opposer un refus à une demande d'information lorsque celle-ci a trait à la sécurité publique. Cette évidence laisse une marge d'interpréta- tion, en particulier pour ce qui concerne l'accès à l'information en période de crise.

L'importance de l'accès à l'information par les citoyens a été reconnue dans le $5^{\mathrm{e}}$ Programme Cadre de Recherche, de Développement Technologique et de Démonstration (PCRD) de l'Union Européenne (1999-2002) [4]. Ce programme vise le développement d'une société de l'information conviviale via un programme spécifique dénommé Programme des Technologies de la Société de l'Information (IST), dont une des actions-clés a pour objectif de stimuler la création d'une nouvelle génération de services d'intérêt général. Des services conviviaux, fiables, peu coûteux et interopérables, répondant aux attentes des utilisateurs qui veulent un accès flexible pour tous, en tout lieu et à tout.

Le Programme IST consiste notamment à :

- Renforcer l'accessibilité, la pertinence et la qualité des services publics dans les domaines de la santé; besoins particuliers, notamment ceux des personnes âgées ou handicapées; administrations; environnement ; transport et tourisme.

- Développer de nouveaux modèles de prestation de services publics.

Notons que dans le domaine de l'environnement de programme IST définit deux axes prioritaires :

- Développement et démonstration, à différentes échelles spatiales et administratives de systèmes et d'outils destinés à une surveillance, au soutien de la planification et de la prévention et à une gestion cohérente de l'environnement au niveau international.

- Développement et démonstration de nouveaux outils et systèmes intégrés pour une gestion cohérente des situations d'urgence tout au long du cycle d'une crise, de la prévention à l'évaluation en passant par l'identification et l'atténuation des effets, qu'il s'agisse de risques d'origine humaine ou naturelle.

Il est clair que l'émergence des échanges en réseau, confortée par les possibilités offertes par les technologies telles que l'Internet et les télécommunications mobiles sont des éléments qui remettent en question les enjeux de pouvoirs liés à l'information. L'intégration des informations prend maintenant le pas sur la "propriété " de l'information [5]. L'important n'est plus de posséder l'information mais de savoir où est disponible l'information fiable. Le progrès technologique a fait sauter le verrou de monopole d'information avec une conséquence essentielle: si une autorité se cramponne à la propriété d'information qu'elle ne veuille livrer qu'aux "experts ", le chemin des décisions de cette autorité sera, en cas de crise, shunté. Et elle perdra la prise sur les événements. L'exemple de la crue de l'Oder nous enseigne que les pratiques spontanées de réseau mises en œuvre entre des citoyens remplissent le vide d'information laissé par des autorités et se substituent aux réseaux officiels quand le contenu de ceux-ci est défaillant. Ces pratiques contribuent à la veille et à l'échange d'informations qui pourraient être utiles aux services de sécurité en temps de crise et devraient en toute logique être intégrées dans le système de gestion de la crise.

\subsubsection{L'émergence du scénario "participatif "- le partenariat des acteurs pour la prévention}

L'instance d'évaluation des politiques publiques consacrées à la prévention des risques naturels [6] appuie ses conclusions sur un scénario d'évolution qualifié de "participatif ".

Celui-ci propose « un partage net des compétences et l'implication dans une politique qui repose sur trois partenaires : l'Etat, les collectivités locales, la société civile ». De ce scénario et des propositions qui s'y rattachent nous retenons la possibilité de complémentarité et de partenariat des acteurs dans le système de prévention. 
Dans un tel contexte, les systèmes mis en œuvre par chaque type d'acteur, loin de s'opposer, sont complémentaires et se renforcent mutuellement.

\subsection{Evolution technologique des systèmes d'annonce et de prévision des crues}

L'évolution des systèmes d'annonce et de prévision des crues est sans doute influencée par le progrès technologique mais en même temps elle est soumise à la réglementation en vigueur.

Rappelons simplement qu'en France l'annonce des crues est actuellement organisée selon un règlement défini dans "L'Arrêté du 27 février 1984 portant réorganisation de l'annonce des crues et de la transmission des avis de crues ». Ces principes reposent sur une clarification des responsabilités et la séparation des processus d'alerte et d'information. La prévision ne fait pas l'objet de ce règlement.

Un nouveau texte en cours d'élaboration, doit cependant tenir compte de l'évolution technologique accomplie depuis 1984.

\subsubsection{Une approche par l'expertise technique}

Historiquement, la mise en place de l'observation systématique des cours d'eau (échelles, réseau d'observateurs, services hydrométriques) a toujours été orientée par l'excellence des moyens techniques (logistiques et méthodologiques). Le développement des systèmes d'annonce et de prévision a tout d'abord été axé sur la mise en place de réseaux de mesures, et depuis 30 ans de réseaux de collecte de données télétransmises, d'outils de modélisation et de prévision en temps réel. Depuis les années 80 et l'apparition des ordinateurs personnels, ces systèmes ont été améliorés par les utilisateurs techniciens, avec la prise en compte de l'ergonomie des relations à l'interface homme-machine.

Pendant la même période en France on a assisté notamment au progrès de la standardisation des réseaux de télémesures en temps réel et à l'installation de tels réseaux dans la majorité des bassins générateurs de crues des cours d'eau importants. Les premières installations intégrées permettant d'effectuer en temps réel la prévision à partir d'un réseau d'acquisition de données ont vu le jour dans quelques services d'annonce de crues (SAC) français. Parallèlement un effort de standardisation des méthodologies de prévision des crues a été entrepris.

\subsubsection{Inondations lentes et rapides}

Les efforts se sont d'abord portés sur l'équipement des grands cours d'eau générateurs de «crues de plaines ». Pour ces crues relativement lentes, les réseaux télémétriques de pluviomètres et de limnimètres, d'une densité raisonnable, permettent d'acquérir en temps réel les données nécessaires pour décrire correctement leur genèse ainsi que la propagation de la crue le long des cours d'eau.

En ce qui concerne les " crues-éclairs " les phénomènes générateurs de catastrophes correspondent à des durées de quelques heures. Ce sont en général des pluies fortement convectives, orages ou successions d'orages, spatialement très hétérogènes et sur des superficies très faibles (Nîmes 1988, Privas 1990, Avignon et Orange 1991, Vaison-LaRomaine et Ardèche 1992, Marseille 1993, etc...). La densité habituelle des pluviomètres installés sur ces bassins versants est souvent insuffisante pour percevoir et décrire ces phénomènes pluvieux aux conséquences catastrophiques. La difficulté d'appréhender les phénomènes à l'aide de réseaux classiques de mesure (pluviomètres et limnimètres), et l'inexistence des mesures de débits en aval des bassins versants concernés rendent évidemment encore plus difficile la création de systèmes de prévision fondés sur l'association mesures/modélisations. De plus, l'expertise du prévisionniste est entièrement conditionnée par les délais très courts d'évolution du phénomène, et l'apport d'outils de synthèse et d'aide à la décision a été nécessaire. Pour toutes ces raisons les Services d'Annonces de Crues ne peuvent pas aujourd'hui assurer d'une manière opérationnelle la prévision des crues-éclairs, pourtant les plus meurtrières, d'où des efforts du Ministère de l'Aménagement du Territoire et de l'Environnement (MATE) visant à combler cette lacune.

Dans cette démarche, afin de pouvoir mieux appréhender la mesure des précipitations, la mise en place de radars complémentaires au réseau ARAMIS et l'installation du METEOTEL dans les SACs ont été entreprises. Des actions pilotes ont été engagées avec pour objet la construction et la validation du prototype d'un outil intégré « Mesures-Modélisation-Radar " [7]. Cet outil devrait permettre, sur les bassins versants de moins de $2000 \mathrm{~km}^{2}$, de quantifier la pluie et d'en modéliser ses conséquences hydrauliques avec une précision et un délai permettant la prévision des crues-éclairs. Des développements similaires ont eu lieu en Europe (par exemple System HYRAD [8]) et aux Etats-Unis (AHPS, NOAA).

Ce développement par l'expertise et la technologie a été facilité par l'offre compétente et structurée de l'Ecole française de l'Eau, qui a trouvé écho auprès d'une maîtrise d'ouvrage à forte culture technique. Il se caractérise par un haut degré de technicité des approches théoriques et de la technologie, par la concentration de l'expertise et des investissements, ainsi que par une certaine centralisation dans la conception même de la mission d'annonce des crues : l'information est maîtrisée par un minimum d'agents compétents.

Néanmoins on constate qu'en France on s'intéresse de plus en plus à la transformation de la prévision concernant les situations à risques en information nécessaire pour les administrations impliquées dans la lutte contre les inondations et la pollution des eaux et à la diffusion de cette information via des réseaux spécialisés aux services de l'Etat, mairies, sécurité civile (réseaux mobiles) ou via Internet vers un plus large public. Par exemple, le Service d'Annonce des Crues du bassin de la Dordogne met à la disposition des services de l'Etat et des élus les informations sur évolution du niveau des eaux sous forme d'un serveur minitel 3614 CRUDOR. L'ensemble de ce système d'élaboration et de diffusion de l'information sera considéré comme un complément indispensable aux systèmes de prévision qui fournissent une information brute pas toujours exploitable et pas toujours tournée vers l'utilisateur final (fig. 3).

\subsection{Evolution de la prévision, de l'alerte et de la ges- tion de crise à l'échelle globale et locale : des initia- tives plus proches du terrain local et du citoyen}

Un certain nombre d'initiatives de terrain montrent une réelle évolution pour apporter des réponses aux besoins locaux d'information.

Nous en citerons deux : la première se rapporte à la démarche de valorisation par les communes de l'information diffusée par les services d'annonce des crues, pour la prise de décision en période d'inondation. La seconde concerne l'alerte personnalisée.

\subsubsection{La démarche de valorisation par les communes de l'information diffusée en période de crue}

Cette démarche [9], mise en œuvre avec le soutien de la Direction de l'Eau du MATE et la participation de la Direc- 


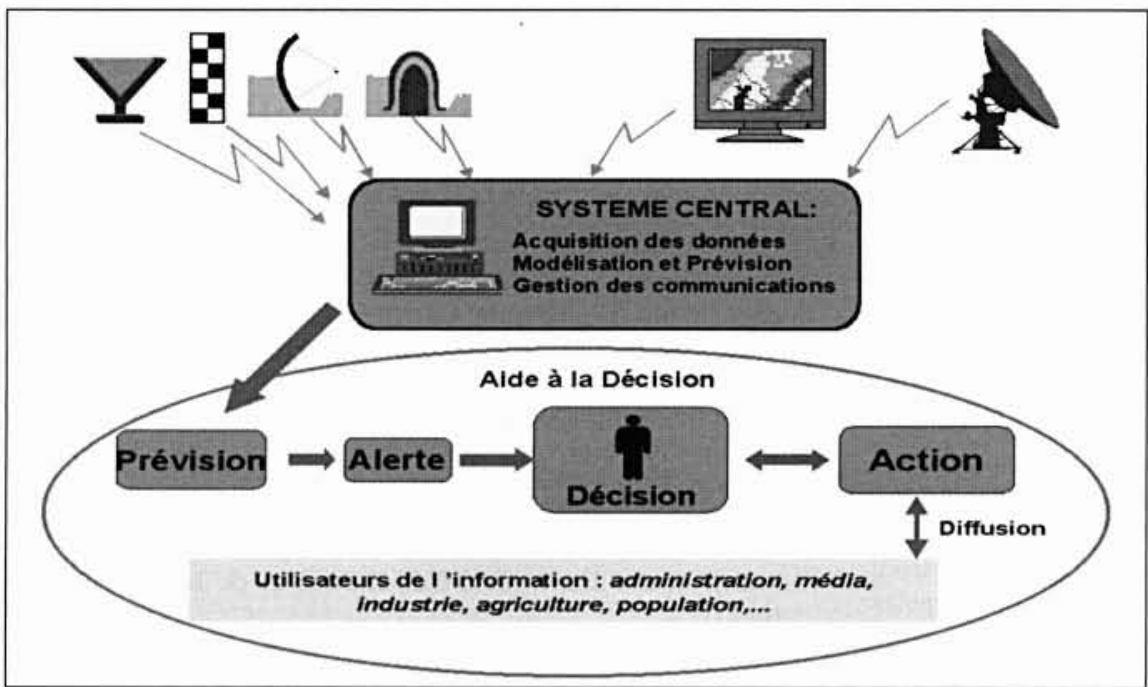

3. Un exemple de l'architecture fonctionnelle d'une plate-forme informatique-télématique pour la prévision des crues et pour la diffusion de l'information en temps réel.

tion de la Défense et de la Sécurité Publique du Ministère de l'Intérieur a pour objectif de donner aux communes les moyens de personnaliser (valoriser), pour leurs propres besoins, des informations transmises par les services de l'Etat dans le cadre réglementaire de l'annonce des crues.

Elle donne lieu à l'élaboration d'outils simples permettant la prévision locale des crues et des inondations, la gestion des interventions, le recueil et l'analyse des informations historiques telles que : la validation du processus de transmission de l'alerte au regard des enjeux locaux, des abaques de corrélation, des échelles de crues locales, des graphes pour la prévision de l'inondation locale, la cartographie des secteurs inondables dès les premiers débordements, la cartographie des prévisions et du suivi de l'inondation..

Orientée sur la logique de service à l'usager, elle articule de manière indissociable quatre composantes: le réseau d'acquisition et de transmission des données, l'expertise hydraulique, la connaissance des conditions locales et une dimension de coopération et d'animation. Le principe consiste à organiser un partenariat entre experts et acteurs locaux afin de mettre au point des outils qui permettront aux communes de réaliser des actions locales de prévention adaptées à la situation hydrométéorologique. L'animation des acteurs est assurée par les services de l'Etat.

Une fois l'enjeu de la coopération et de la valorisation locale des informations maîtrisé, il paraît évident que l'étape suivante consiste à mettre l'information à la disposition des riverains.

C'est pourquoi le développement actuel de la démarche de valorisation de l'information [10] mené dans le cadre de la modernisation du système CRISTAL par l'EPALA et la DIREN Centre sur le bassin de la Loire donne également lieu à une expérimentation qui vise la mise à disposition permanente des informations relatives au niveau de la Loire.

L'utilisation d'Internet, par le biais des serveurs municipaux, permet d'organiser la mise à disposition d'informations générales concernant les risques, mais aussi la communication en période de crue grâce à la consultation des prévisions locales de l'inondation des secteurs de la commune. La réalisation d'Auvers-sur-Oise (http://www.auvers-sur-oise.com), qui fut l'une des premières communes associées au développement de la démarche de valorisation de l'information, paraît exemplaire de ce point de vue.

La démarche de valorisation de l'information constitue une réponse au besoin d'information des élus et riverains, que ce soit à titre préventif ou en période de crise. Elle associe de manière participative le niveau global (de niveau bassin ou sousbassin, tronçon de rivière) et le niveau local (commune, quartier, secteur).

\subsubsection{Alerte personnalisée}

Les évolutions technologiques permettent, en automatisant l'alerte, d'en améliorer les conditions d'émission (appels simultanés) et de réception. Le système d'alerte par automate D.A.L.I. mis en œuvre par la Direction de la Défense et de la Sécurité Civile permet ainsi d'automatiser l'alerte réalisée dans le cadre réglementaire vers les maires ou les représentants que ceux-ci ont désignés.

Il est ensuite du devoir du maire d'alerter à son tour ses concitoyens exposés au risque annoncé. Différents moyens sont employés pour ce faire, à portée collective (de type sirène, voire grâce à la télévision ou à la radio) ou individuelle (porte à porte, contact téléphonique personnalisé, appel par automate). Nous retenons encore ici l'apport de la technologie qui permet, comme c'est le cas sur la commune de Bollène, d'alerter individuellement chaque riverain préalablement recensé.

Ces différents moyens sont complémentaires plutôt que concurrents. En effet l'alerte individuelle par automate présente l'intérêt de toucher le destinataire en tous lieux et en tous temps grâce à la téléphonie mobile.

L'alerte n'est pas une fin en soi. Donnant lieu à une action de sauvegarde, elle doit être associée à des consignes de comportement d'autant plus élaborées et compréhensibles que l'alerte est performante.

\subsection{Vers un système d'information pour l'aide à la décision et l'intervention}

Ces éléments permettent d'envisager pour les prochaines années l'apparition de systèmes aux fonctions complémentaires aux systèmes d'annonce et de prévision existants. Nous imaginons que ces systèmes d'information sur la situation dans le bassin pourront répondre à un scénario basé sur un service individualisé, compatible avec les exigences de la sécurité collective et avec des principes de responsabilisation, d'entraide et de participation des citoyens.

\subsubsection{Réseaux de mesures}

Les réseaux de mesures ne seront plus dédiés à une fonction déterminée (telle l'annonce des crues par exemple). On utilisera le principe de la mise en réseau des réseaux de stations automatisées, ce qui permettra de disposer des informations complémentaires et d'un fonctionnement relativement satisfaisant en conditions dégradées. A chaque station hydrométrique pourra être associé un coefficient de pertinence au regard de chaque critère (indicateur qualité, crue, étiage).

Les réseaux "à vocation multiple "serviront les besoins d'information pour différentes fonctions et différents gestionnaires : gestion du risque naturel, suivi de la qualité des eaux, connaissance patrimoniale, gestion de barrages, assainissement et eau potable. 
Les gestionnaires de réseaux développeront une nouvelle fonction, axée vers la valorisation des données. Elle consistera à mettre en œuvre des organisations, méthodes et outils permettant d'utiliser au mieux les données acquises par les réseaux afin de satisfaire les besoins en informations des riverains, citoyens, acteurs économiques, collectivités locales. L'accès à cette information sera rendu possible aux citoyens à partir des terminaux portables.

\subsubsection{Annonce et prévision des crues}

Les crues faisant l'objet de prévisions bien maîtrisées et fiables, l'effort sera porté sur la gestion des conséquences. L'usage des SIG permettra de représenter la prévision et le suivi des inondations sur plans à l'échelle locale et globale. Les techniques de prévision étant maitrisées par utilisation des données multi-sources et des modèles qui permettront avec précision d'établir la cartographie des zones inondables en tenant compte de la variabilité spatiale de l'humidité des sols, les services d'annonce des crues porteront leurs efforts sur le contact de terrain avec les représentants locaux : élus, services d'intervention, riverains, pour mieux connaître les enjeux locaux, responsabiliser et définir les informations et outils permettant d'améliorer la prévision et l'intervention locales.

\subsubsection{Gestion de crise}

L'étude préalable de scénarios de crise en fonction des conséquences locales de l'inondation permettra de prévoir les moyens pour parer aux inondations, des plus courantes aux plus catastrophiques selon une typologie bien déterminée. Les résultats de ces scénarios établis hors ligne seront stockés dans une base de données et exploités en période de crise pour trouver les solutions les plus adaptées à la situation.

Les principes de l'organisation en réseau permettront d'associer les moyens locaux aux structures centrales de gestion de crise, en particulier pour l'intégration des données de terrain aux scénarios initiaux pour l'intégration et le développement de systèmes spontanés d'entraide associés aux systèmes professionnalisés.

La mission des gestionnaires de la sécurité civile, associés aux services de secours et d'incendie, se développera vers la pédagogie. Des échanges avec les collectivités locales permettront un apport de savoir-faire pour l'élaboration de plans de secours locaux. Les bases de données de vulnérabilité locale pourraient être renseignées par les collectivités locales et services d'intervention sous l'impulsion des services de sécurité civile.

\subsubsection{Interaction avec les citoyens}

Ainsi la prise en compte des problématiques locales passe par une approche impliquant les acteurs locaux eux-mêmes dans le processus d'élaboration et d'adaptation de l'information diffusée par les services d'annonce des crues. Cela sousentend une démarche coopérative demandant le management d'interrelations entre services, écoute mutuelle dans le cadre d'équipes de cultures différentes avant, pendant et après la crise.

La diversité des situations de terrain suggère la prise en compte et la valorisation de cette diversité plutôt que l'uniformisation des méthodes et des solutions, ce qui tend à faire émerger des logiques de fonctionnement en réseau plutôt que des logiques hiérarchiques et centralisatrices.

\section{III —VER DES SOLUTIONS OPÉRA- TIONNELLES POUR LA GESTION DES RISQUES D'INONDATION DANS LA SOCIÉTÉ DE L'INFORMATION - LE PROJET OSIRIS}

Un groupement de partenaires européens a récemment proposé, dans le cadre de l'action clé Systèmes et services pour le citoyen du programme thématique "Société de l'information conviviale " (IST) évoqué ci-dessus, le projet OSIRIS («Operational Solutions for the management of Inundation Risks in the Information Society »). OSIRIS s'intéresse à l'amélioration des systèmes et procédures de lutte contre les inondations dans le cadre d'une société qui vit une accélération des applications basées sur les Nouvelles Technologies de l'Information et de la Communication (N.T.I.C).

OSIRIS considère toutes les phases de la gestion du risque d'inondation, depuis la prévention, la préparation de plans d'intervention, la surveillance de la rivière et la prévision de ses excès (phase préparatoire), l'alerte, la mise en place de mesures d'atténuation des effets de la crue, la gestion de la situation de crise jusqu'au suivi de ses conséquences et le rétablissement d'une situation normale.

Les objectifs généraux du projet concernent ces différentes phases. Ils consistent à :

- Les analyser à différentes échelles spatiales aussi bien dans les grands bassins versants fluviaux que dans les zones urbaines qui les bordent.

- Améliorer leur cohérence et leur efficacité, en s'appuyant sur les nouvelles technologies de l'information, du point de vue de la transmission des flux d'information, de la prise de décision et de la gestion de la crise.

Un soin particulier sera accordé à la sensibilisation de la population occupant les zones à risque par la mise en place de moyens simples, mais efficaces, qui permettront de développer la culture du risque d'inondation.

OSIRIS reçoit le support de l'Union Européenne de l'Etablissement Public d'Aménagement de la Loire et de ses affluents, et du Centre d'Etudes Maritimes et Fluviales du ministère de l'Equipement, des Transports et du Logement.

\subsection{Un projet orienté vers l'implication du citoyen dans la gestion du risque d'inondation}

Les systèmes actuels de gestion du risque d'inondation sont en général plus dédiés aux organismes centraux gestionnaires de crise (autorité de bassin, administration, protection civile,...) qu'orientés vers les populations riveraines des cours d'eau et leurs représentants. Il existe donc à l'heure actuelle un besoin très fort de systèmes d'information et de procédures orientés vers la satisfaction des besoins d'information et d'intervention des riverains citoyens et de leurs représentants, ce à toutes les phases de la gestion du risque d'inondation (phase préparatoire, crise elle-même et situation post-crise). OSIRIS se propose de satisfaire ces besoins au travers d'objectifs consistant à :

- Sensibiliser le citoyen sur le risque d'inondation et accroître son implication dans les processus de prévention et de gestion de crise.

- Préparer les citoyens et les gestionnaires de crise à des actions de protection et de secours efficaces en période de crise.

- Améliorer la qualité de l'information fournie aux parties prenantes du risque d'inondation (gestionnaires de bassin, protection civile, citoyens et leurs représentants) avant, pendant et après la crise. 
- Accroître la rapidité et la flexibilité d'accès à l'information grâce à l'utilisation de nouveaux supports de transmission et de distribution de l'information, ainsi que grâce à la mise à disposition d'une information élaborée à tous niveaux (central ou local) et en tous lieux.

Le partenariat du projet OSIRIS est composé d'une association de 12 organismes appartenant à 5 pays européens (France, Allemagne, Pologne, Italie, Pays Bas). Ces partenaires ont des statuts et des cultures très diverses, mais tous sont intéressés par l'amélioration de la gestion du risque d'inondation. Il s'agit d'une équipe pluridisciplinaire, composée de spécialistes en systèmes de prévision de crue, en organisation et gestion, en informatique, de sociologues et d'économistes. Ce partenariat est structuré en trois groupes où sont représentés des utilisateurs finaux (y compris les décideurs en situation de crise), des chercheurs et des consultants :

Gestionnaires de bassins hydrographiques et représentants du citoyen (municipalité ou syndicat de communes) :

- La Direction Régionale de I'Environnement - DIREN - CENTRE et l'Etablissement Public d'Aménagement de la Loire et de ses affluents - EPALA - (France).

- La ville de Francfort/Oder (Allemagne).

- L'Institut de Météorologie et de Gestion de l'Eau (Pologne).

Organismes de recherche :

Le Centre d'Etudes Techniques Maritimes et Fluviales CETMEF - du Ministère de l'Equipement des Transports et du Logement (France).

- L'Institut International d'Ingénierie en Infrastructures, Hydraulique et Environnement, Delft (Pays Bas).

- L'Université Technologique de Cottbus, Brandenburg, (Allemagne).

- L'Institut de Psychologie de l'Académie Polonaise des Sciences, Varsovie (Pologne).

Sociétés de services :

- SOGREAH, Grenoble (France), coordinateur du projet.

- Guy TALIERCIO Consultants, Grenoble (France).

- Economie et Humanisme, Lyon (France).

- Analysis Automation Trading Srl, Florence (Italie).

Les sites tests retenus dans le cadre du projet concernent deux bassins hydrographiques :

- Le bassin de la Loire: Loire moyenne.

- Le bassin de l'Oder (Odra) : le cours moyen de l'Oder avec la ville de Francfort sur Oder en Allemagne et la ville de Slubice/Odra, située sur la rive droite du fleuve en Pologne, et le cours de la Nysa Klodzka dans le bassin supérieur de l'Odra, commune de Klodzko.

Le choix de ces deux sites et l'implication des utilisateurs finals de chacun d'entre eux confirme l'envergure européenne du projet. En particulier, OSIRIS mettra en valeur le retour d'expérience de la crue de l'Odra (Oder) en juillet 1997 dans tous ses aspects, au profit des gestionnaires d'autres bassins en Europe : analyse des défaillances du réseau de communication, des mesures de terrain, de gestion de la crise avant, pendant et après l'événement catastrophique qui a dépassé de 2,5 fois la crue centennale du fleuve. Cette coopération entre les techniciens du Bassin de la Loire et leurs collègues polonais a d'ailleurs déjà été engagée bien avant la crue de 1997.

\subsection{Un projet résolument opérationnel}

Les objectifs du projet seront atteints par les actions principales suivantes:

- La définition et le test d'actions participatives pédagogiques visant à développer la prise de conscience des citoyens sur le risque d'inondation et la prise d'initiatives en période de crise ;
- La définition et le test de scénarios de crise et de plans d'action d'urgence visant à protéger les personnes et les biens ;

- La conception et le développement de prototypes de Systèmes de Gestion de l'Information et de Communication dédiés (1) aux gestionnaires de bassin, (2) aux centres de secours opérationnels, (3) aux citoyens et à leurs représentants. Ces systèmes seront basés sur l'utilisation des nouvelles technologies de l'information et de la communication (téléphonie mobile, INTERNET,...). Leur vocation consistera à :

- Transformer l'information sur les risques d'inondation (observations de terrain et vulnérabilité des biens, données de surveillance et de prévision,...) en une information synthétique, facilement compréhensible, adaptée aux différentes administrations impliquées dans la prévention et la gestion de crise, mais aussi aux populations riveraines. Différents niveaux d'éducation du public seront considérés. Le niveau approprié de contenu d'information et de représentation de celle-ci sera atteint à l'aide des technologies récentes de traitement de données (Systèmes d'Information Géographique, systèmes à base de connaissance, interfaces graphiques, modèles numériques, etc.).

- Mettre à disposition cette information, pour le cycle complet de gestion des risques d'inondation, à toutes les parties prenantes (gestionnaires de la crise, services de protection civile, citoyens), grâce aux médias appropriés.

- Développer des méthodes de contrôle de la propagation des imprécisions et incertitudes sur les données mesurées et les informations de base tout au long de la chaîne de traitement de l'information, depuis la source des observations et des décisions humaines jusqu'à l'utilisateur final.

- Mettre au point des systèmes et des méthodes de prévention, de surveillance, d'alerte et de secours cohérents (à l'échelle inter-départementale, inter-régionale, voire internationale).

- Etablir des recommandations aux gestionnaires de bassins ainsi que des propositions pour un futur standard européen couvrant les aspects législatifs et organisationnels, les plans d'action en situation d'urgence, la prévention et la gestion du risque, d'inondation en général.

- Diffuser aux parties prenantes de la gestion de crise et aux scientifiques les aboutissements du projet, et ce à un niveau européen.

Le programme de travail se décompose de la façon suivante :

A. Le retour d'expérience : collecte auprès des organismes impliqués dans la gestion de crise (utilisateurs finals) des informations concernant notamment les aspects sociologiques, réglementaires et techniques pour la gestion des inondations au travers de situations vécues. L'implication de différents pays de l'U.E. dans le projet permettra de disposer d'un état de l'art représentatif au niveau européen.

B. Le recensement des besoins en matière d'information : ce recensement sera fait en tenant compte des expériences passées mais également en explorant toutes les possibilités offertes par les nouvelles technologies, méthodologies et concepts qui pourront être développés en termes d'acquisition et de diffusion de l'information. En pratique, des enquêtes seront réalisées sur les sites pilotes, auprès des différentes classes d'utilisateurs finals (gestionnaires de bassin, services de sécurité civile, citoyens et leurs représentants,...).

C. La définition des systèmes et des stratégies : à partir des éléments recueillis lors de l'étape précédente, seront définis les systèmes d'acquisition et d'alerte, de prévention, de prévision, les stratégies de gestion de crise et d'aprèscrise et les systèmes d'information qui devront être mis en place. Cette définition sera faite d'abord à un niveau conceptuel ; elle sera ensuite adaptée à chaque site pilote du projet. D. Le développement et l'implantation : les systèmes de gestion de l'information et de la communication seront déve- 
loppés (sous forme de prototypes) et vérifiés. Ils seront implantés sur chacun des sites pilotes.

E. Démonstration et retour d'expérience : les systèmes de gestion de l'information et de la communication seront testés grâce à des exercices de simulation sur le terrain à petite échelle; on en tirera les premières leçons de l'utilisation du système. Ces expérimentations pourront être effectuées au travers des crues qui pourront se produire durant cette phase ou de la simulation de scénarios de crise définis précédemment. Cette expérimentation permettra, par le retour d'expérience, d'améliorer le fonctionnement des systèmes proposés.

F. Généralisation : cette étape permettra de tirer les enseignements des résultats obtenus dans le cadre du projet et de définir des recommandations utilisables au niveau européen pour l'élaboration d'une réglementation européenne en matière de gestion des inondations.

En complément de ces différentes étapes, diverses actions de diffusion des résultats du projet et d'échanges sont prévues grâce à la création d'un site INTERNET du projet, l'organisation de forums d'utilisateurs sur INTERNET et l'organisation d'une conférence en fin de projet sur la gestion des inondations, ainsi que la participation à différentes manifestations scientifiques et techniques.

En France, OSIRIS présente l'opportunité de réaliser une plate-forme opérationnelle, d'envergure expérimentale, complète sur le Bassin de la Loire. Celle-ci intégrera et équipera le service de bassin, la protection civile, les services d'intervention, les maires et les riverains de communes pilotes. S'agissant d'un projet de démonstration, cette plateforme permettra à l'EPALA et à la DIREN CENTRE d'aller plus loin dans l'axe de leurs travaux concernant l'organisation et les moyens au service de l'usager.

\section{CONCLUSIONS}

En Europe, certaines des évolutions évoquées dans cet article sont déjà engagées par les services de l'Etat. D'autres, font partie de projets récents, comme OSIRIS

L'expérience de la crue de l'Oder a montré la volonté et la capacité des citoyens à participer à la gestion de la crise. Elle a illustré le fait que le "verrou " de la propriété et du partage de l'information est difficile à ouvrir.

Alors quelles seront les limites à la faisabilité d'un scénario " participatif » qui permettrait aux Etats de développer une initiative tenant compte des besoins et capacités de la société civile et des collectivités locales dans la société de l'information? Enrichis des résultats du projet OSIRIS nous espérons donner une réponse à cette question dans les 3 ans à venir.

\section{RÉFÉRENCES}

[1] Rapport de la Commission d'enquête de l'Assemblée Nationale (1994). - «Inondations : une réflexion pour demain ", Philippe Mathot (Président), Thierry Mariani (Rapporteur). Députés. Rapport n 1641. D.I.A.N. 58/94, 2 tomes.

[2] Loi du 22 juillet 1987 d'organisation de la sécurité civile et de prévention des risques majeurs. Décret d'application du 11 octobre 1990 relatif à l'exercice du droit à l'information sur les risques majeurs.

[3] Directive 90/313/CEE du Conseil, du 7 juin 1990, concernant la liberté d'accès à l'information en matière d'environnement.

[4] Commission Européenne (1999), Appel de propositions pour des actions indirectes de RDT dans le cadre du programme spécifique de recherche, de développement technologique et de démonstration intitulé «Créer une société de l'information conviviale (1998 à 2002) ", (Programme des technologies de la société de l'information, Bruxelles, le 16 mars 1999.)

[5] Commission Française du Développement Durable (1996) - Rapport : «Contribution au débat national sur le Développement Durable - Priorités d'action ", p. 40.

[6] Rapport de l'instance d'évaluation présidée par Paul-Henri Bourrelier (1997), - « La prévention des risques naturels ", La Documentation Française, novembre 1997.

[7] Cunge J., Erlich M. et Rahuel J.L (1998), - « Expérience du LHF dans le développement et transfert international des technologies avancées dans le domaine de la

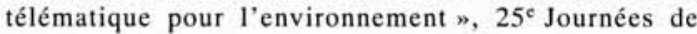
l'Hydraulique, Chambéry, SHF, Houille Blanche 3-4, pp. 61-67.

[8] MoORE R.J. (1994), - Integrated systems for the hydrometeorological forecasting of floods. Verri (ed.): Proc. Intl. Scientific Conference EUROPROTECH, Part I : Science and technology for the reduction of natural risks, May 6-8 1993, CSIM, Udine, Italy, pp. 121-137.

[9] Taliercio G., Le Trionnaire Y., Bouysses H., Godon C. Odier M., Roy J.L. (1998), - Améliorer la prévision locale des inondations : une démarche pragmatique de valorisation par les communes de l'information diffusée par les services d'annonce des crues. L'Ecole française de l'eau au service du développement mondial, $25^{\mathrm{c}}$ Journées de l'Hydraulique de SHF, Chambéry, 15-18/09/98, T. 1, p. 257

[10] Taliercio G., Moulin L. (1999). - Expérience de valorisation de l'information pour la prise de décision en période de crue et en période d'étiage. Colloque de la SHF, Paris, 29 et 30 septembre 1999, pp. 233-236. 